\title{
A Data Integration And Analysis System For Sea Ice Remote Sensing
}

\author{
Zhang, H.; Pedersen, Leif Toudal; Skriver, Henning; Gudmandsen, Preben
}

Published in:

International Geoscience and Remote Sensing Symposium

Publication date:

1991

Document Version

Publisher's PDF, also known as Version of record

Link back to DTU Orbit

Citation (APA):

Zhang, H., Pedersen, L. T., Skriver, H., \& Gudmandsen, P. (1991). A Data Integration And Analysis System For Sea Ice Remote Sensing. In International Geoscience and Remote Sensing Symposium: Remote Sensing: Global Monitoring for Earth Management (Vol. Volume 2, pp. 787-790). IEEE.

\section{General rights}

Copyright and moral rights for the publications made accessible in the public portal are retained by the authors and/or other copyright owners and it is a condition of accessing publications that users recognise and abide by the legal requirements associated with these rights.

- Users may download and print one copy of any publication from the public portal for the purpose of private study or research.

- You may not further distribute the material or use it for any profit-making activity or commercial gain

- You may freely distribute the URL identifying the publication in the public portal

If you believe that this document breaches copyright please contact us providing details, and we will remove access to the work immediately and investigate your claim. 


\title{
A DATA INTEGRATION AND ANALYSIS SYSTEM FOR SEA ICE REMOTE SENSING
}

\author{
H. Zhang, L.T. Pedersen, H. Skriver and P. Gudmandsen \\ Remote Sensing Unit, Electromagnetics Institute, Technical University of Denmark \\ Building 349, DK-2800 Lyngby
}

\begin{abstract}
With the advent of ERS-1 and later microwave satellite missions the amount of data acquired for sea ice research and monitoring becomes very large. To obtain the full benefit from this it will be necessary to systematize the great amount of data obtained and to exploit the knowledge and the analysis methods developed in the past. For this purpose, a data integration and analysis system is being developed that may accommodate all types of remote sensing data as well as other data to be used in modelling of the air-sea-ice interaction processes. This paper presents the system with emphasis on system requirements, design and development of a database. Various parameter retrieval algorithms are briefly described.
\end{abstract}

Keywords: Sea ice remote sensing, data integration and processing

\section{INTRODUCTION}

Various remote sensing data have been used successfully for extracting useful information for sea ice research and monitoring. However, various types of sensor have different capacities and systematic shortcomings. Thus, major difficulties in using data from passive visual, infrared and microwave remote sensors for studies of polar regions are the susceptibility to clouds in case of the visible and infrared sensors, and the rather coarse spatial resolution of the microwave sensors. It is expected that a great deal of valuable information will be obtained from the ERS-1 SAR data, due to the fine spatial resolution and the independence of light and cloud condition of this type of data. But due to the limited sampling in time and space even in subpolar oceans like the Greenland Sea, the data are believed to be complementary to traditional data sets which form the basis of the present sea ice observations. Supports of non remote sensing observations such as buoy drift data and meteorological data are also needed. Therefore, to carry out a comprehensive study of sea ice processes, data acquired from various observation methods will complement each other, and for this purpose, a system of data integration is likely to be of advantage. In addition, in order to be able to cope with the very large amount of data of sea ice observations a set of automatic or semi-automatic analysis algorithms is needed, supported by a powerful database system.

A retrieval of sea ice parameters (and other geophysical parameters) from remotely sensed data is to a large extent based on à priori knowledge about the geophysical situation and selection of suitable parameter retrieval algorithms. Such knowledge resides primarily with human interpreters or is stored in various data formats in various places. For optimum data interpretation a comprehensive information extraction system is required which is coupled to an automatic or semi-automatic methodselecting capability whereby the task of the interpreter is reduced.

In summary, in order to carry out studies of sea ice more efficiently, it will be advantageous to use an analysis system with the objectives:

\footnotetext{
- of integrating various types of data and handling of a large and increasing amount of data;

- of systematizing the knowledge on the sea ice research obtained in the past, i.e. to set up a knowledge base,

- and various algorithms of parameter retrieval;

- of including a set of highly automated, flexible and powerful image processing functions
}

A data integration and analysis system with these objectives is being developed. As a pilot project, the work presented in this paper focus primarily on defining and outlining briefly the sys- 
tem scheme, the functions and requirements of each subsystem, the development of algorithms for ice parameter extraction from remote sensing data and the development of a database system and various types of interfaces to connect the database with other parts of the system and application programmes. The system is considered an end system, and only part of it has been implemented today.

\section{SYSTEM FUNCTIONS AND SCHEME}

To achieve the objectives outlined in the introduction, the data integration and analysis system should combine the functions of a general remote sensing image processing system with algorithms to retrieve various sea ice and ocean parameters and a database system. That is, the system should be capable of providing

1. a database management to facilitate storage, search and combination of a substantial amount of data from a variety of sources, so that scientists will be able to retrieve and integrate various data into parameter retrieval and remote sensing image interpretation processes;

2. a set of pre-processing and processing tools for processing of remotely sensed data;

3. a set of retrieval algorithms to extract sea ice and related parameters;

4. a combined display capability to visually show the input, output and intermediate results of data processing and parameter retrieval;

5. a user friendly interface supporting both novice and expert users so that any scientist can express his/her ideas easily and carry out the data processing and parameter retrieval operations;

6. a set of input and output facilities to convert data of various formats and/or sources to one of pre-defined standard formats of the database, and to output system products in different formats and types.

The system shall include the full spectrum of functions needed for sea ice research. Because of the broad range of data types which the system will deal with and the high capabilities needed in information extraction and data interpretation for sea ice research, a general Geographic Information System (GIS) cannot fulfil the requirements. On the other hand, an extensive search of available general image processing systems revealed none that could meet the stringent requirements by itself without a combination with the information system and parameter retrieval modules.

A system scheme is designed and illustrated in Fig. 1. The system frame to enable easily future growth as needs may arise. It consists of a database, a display sub-system, a pre-processing and processing module, a tool box of various general digital image processing and analysis algorithms, a parameter retrieval module and a set of input and output facilities. A modelling module is also included in the system to provide a simulating capability for algorithm development and data interpretation.

The hardware system consists of an IBM 7350 image processing system hosted by IBM 370 VM machine, an IBM RT-PC 6150 system, a IBM PC/AT, and an IBM PS/2-30. with connecting network.

\section{SYSTEM ELEMENTS}

The work in developing this system is divided into three major parts: development of a remote sensing image processing sys-

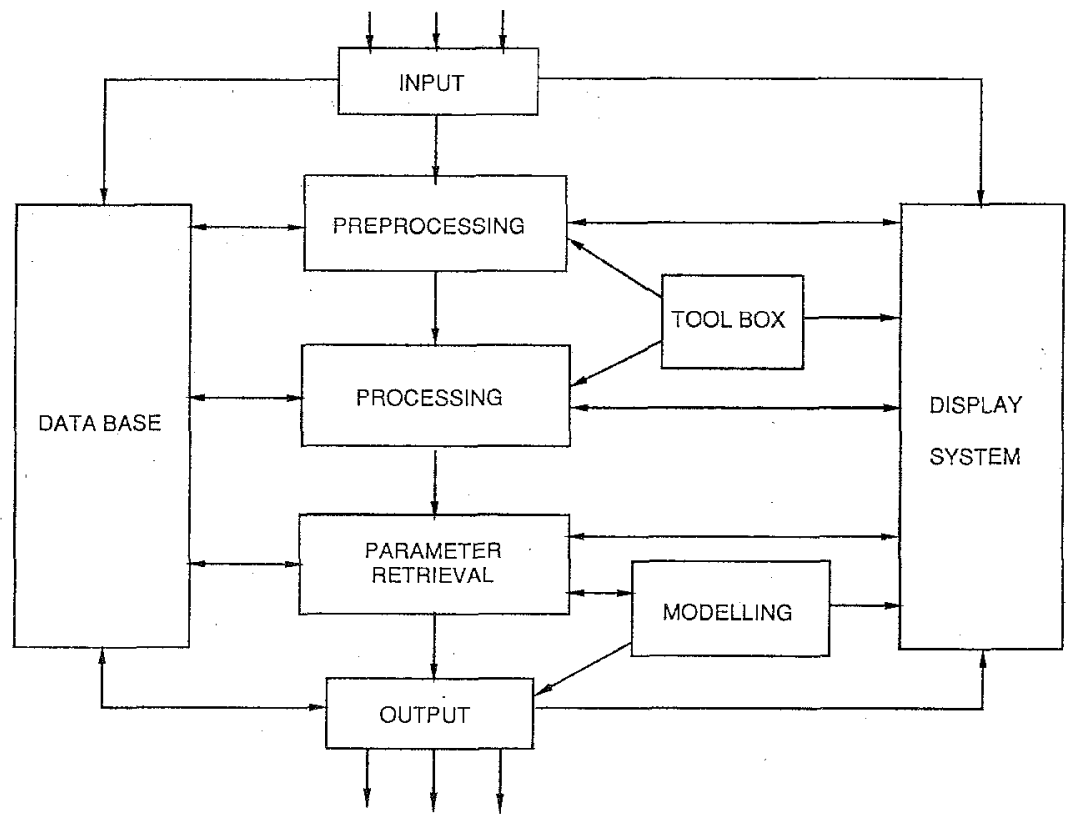

Figure 1:

Schematic diagram of the data integration and analysis system for remote sensing of sea ice. 
tem, development of parameter retrieval algorithms and development of a database system. Input and output facilities are also important parts of the system. The functional requirements and design of each sub-system are as follows.

\section{Database sub-system}

The DBMS (Data Base Management System) is developed to facilitate storage, retrieval and combination of a substantial amount of data from a variety of sources [1]. The data collection in the DBMS includes data from various remote sensing instruments as well as other measurements such as buoy data and data from ground stations. As illustrated in Fig.1, the database is connected to various modules in the system and can also be connected to user's application programmes. The operations that will be carried on by the database include:

1) creation and maintenance of a catalogue of all data currently available within a particular facility. This catalogue contains the necessary information about the data so that it can be retrieved by a set of logical statements

2) insertion of new data into the catalogue as they are acquired, or from the output of processing, for parameter retrieval and modelling;

3) location and combination of data as they are queried to process, for parameter retrieval, modelling or display;

4) control of the archiving digital images on peripheral storage devices (disk and tape);

5) establishment of a catalogue and index of all hard-copy photographic products or image image data which are not stored in the system but on tape or other media.

To achieve these functions, two sets of flexible, efficient and user-friendly interfaces, programmed in C and SQL, have been developed: (a) the menu-oriented interactive user interfaces and (b) the interface functions to be used in connection with the application programmes. Interactive SQL commands and commands embedded in user application programmes are also supported to access database. It has been found that this database satisfies the main requirements of the system although some improvements are needed in order to carry out a more complex data integration.

\section{Image processing sub-system}

Image processing functions are required in support of the parameter retrieval processes. It contains a full suite of algorithms that perform all standard image processing functions in order to rake optimum use of the image data in parameter retrieval and image display. The image processing software systems are designed in a modular fashion including the pre-processing, the processing (tool box) and the display module.

The Display module provides the user with the capability of viewing and manipulating thesactual data in various graphic rep- resentations and help the user to make visual interpretations regarding the information content of data. It has been developed based on the IBM 7350 system. The display module has a userfriendly menu-oriented interactive interface and provide display capabilities required:

1) Image display, including (a) black-and-white and colour display, with the capability that a number of images can be displayed on the same screen in different partitions; (b) grouping pixels by pseuso-colour manipulations; (c) graphic overlay and annotation.

2) Cursor operation, including (a) location handling on displayed image, e.g. in ground control point selection for geometric correction of AVHRR images and selecting tie points for ice concentration estimate, (b) defining features in images, e.g. draw polygons by moving cursor to define an area, and ice feature tracking from a pair of displayed images.

3) Image zooming and interactive Look-Up Table (LUT) manipulation. That is, the user can focus on a subset of an image and can also enhance the features of interest on images displayed.

4) Read-back of images, graphics and the LUT, and save them into files.

The function of the pre-processing module is to convert input data into geometrically and radiometrically corrected images. Accurate geometrical correction of input image is critical in measuring absolute ice motion, for instance. Relative radiometric calibration is important for image segmentation processes, for instance, where consistency is required both across an image and from image to image. The radiometric effects to be corrected include the atmosphere, illumination conditions, sensor calibration, viewing geometry, sun elevation, and the measurement units, e.g, radiance. The geometrical corrections include pixel size variation due to scan-angle effects, registering of images to specified map projection, image to image registering and to compute pixel coordinates. An appropriate algorithm can be selected based on the input information, including: type of instrument and platform, orbit data, sun position, atmospheric conditions, reference data, image control points and performance criteria. When data is input for pre-processing, the database entry associated with the data is generated in this module.

The geometrical correction algorithms for correcting AVHRR image is a combined analytic and synthetic algorithm [2] that uses both orbit parameters and ground control points. A sun elevation correction programme is also implemented in this module. Image construction from SMMR, SSM/I and MSR data is also included in this module.

The Tool Box provides a wide range of general image processing and data manipulation functions necessary for the pre-processing, display and parameter retrieval modules. These include 
retrieve, display, rotate, scale, filter, enhance, save, and generate statistics for image data. Both interactive and batch processing are supported.

The system includes a number of the usual peripherals.

\section{Parameter retrieval sub-system}

The parameter retrieval sub-system provides various algorithms to derive sea ice and related parameters from geometrically and radiometrically corrected remotely sensed images and other types of data. The algorithms implemented include:

- A set of methods for ice motion tracking from consecutive scenes of remote sensing data, including a computer-assisted ice feature tracking algorithm, an area-based correlation algorithm and an automated ice-floe segmentmatching algorithm [ 3].

- Algorithms to determine total ice and ice type concentrations from passive microwave data (SMMR and SSM/I) as well as methods for deriving ocean and atmospheric parameters [4].

- Algorithms for determining ice parameters from SAR data using various statistical tools (feature vectors including texture measures) and image processing techniques [5].

- A split-window algorithm for determining sea surface temperature from infrared data [6].

These parameter retrieval processes can be run in both interactive and batch mode. In the interactive mode, a menu oriented input/output interface is provided. The system frame is designed in such a way that a scientist can choose the proper algorithms under advice given by the system. This capability is very important due to the fact that a specific parameter retrieval in general is developed based on a specific types of remotely sensed data for a given geophysical situation. In order to provide sufficient and proper advice to users, a knowledge base is defined in the way that if conditions then the most suitable algorithms and procedures are selected. Thus, users only need to specify the relevant parameters, and the system will be able to provide an optimum choice based on the knowledge included.

\section{FUTURE WORK}

With the system presented above, the required data integration and analysis capabilities have been achieved, although presently limited by both computer facilities and developing time.

To improve the capabilities of image and graphic display and processing as well as system portability, a more powerful work station is needed to replace the function of the IBM RT PC and 7350. That is, the entire data integration analysis system will be based on the work station rather than a host main-frame system. A necessary future improvement will be to reduce the level of human efforts required for the analysis without compromising performance
In order to provide for automatic processing and analysis for operational applications, an improvement could be to use a production schedule as described in [7]. When a data set is received by the system, a pre-defined production procedural schedule, determined by the geographic region, the season and time is called upon to operate on the input. Parameter retrieval processes can be improved by using artificial intelligence (AI) techniques, such as neural network for classification and automated pattern recognition. Knowledge-based techniques can be used for automated algorithm selection.

\section{CONCLUSIONS}

A data integration and analysis system for sea ice remote sensing has been developed under the Greenland Sea Project with applications focusing on the Greenland Sea. The system has been used to derive data of various ice parameters for the Greenland Sea. From the applications the system is shown to be able to provide users with the means of combining the functions of a general image processing system, a database and a set of parameter retrieval algorithms, so that a substantial amount of data can be retrieved, processed and integrated into information extraction and remote sensing image interpretation processes. In this way, the efficiency of scientists in determining geophysical parameters is improved. An example of the use of the system implemented so far is described in [8].

\section{REFERENCES}

[1] H. Zhang, "A database for an analysis system of sea ice observations", Report of Greenland Sea Project, 80 pp., in press.

[2] P. Hansen, "A combined analytic and synthetic method for geometric rectification of satellite imagery", Report of the Electromagnetics Institute, R290, 60 pp. 1984.

[3] H. Zhang, "Sea ice tracking algorithms", Report of the Greenland Sea Project, No.33, 46 pp. 1990 .

[4] L. T. Pedersen, "Retrieval of sea ice concentration by means of microwave radiometry", Ph.D Thesis, $\mathrm{LD} 81$, Electromagnetics Institute, 1991, 148 pp. 1991.

[5] H. Skriver, "Extraction of sea ice parameters from synthetic aperture radar images", Ph.D Thesis, LD 74, Electromagnetics Institute, 335 pp. 1990.

[6] S. Kristensen, "Sea surface temperature algorithm", Report of Greenland Sea Project, No. 24, 25 pp, 1989.

[7] B. Ramsay, D. Henderson, and L. Carson, "Real-time processing of digital image data in the support of the Canadian sea ice analysis and prediction programme", Proc. IGARSS'88 Symp., pp. 1707-1711, 1988.

[8] H. Zhang and P. Gudmandsen, "Relations between sea ice motion derived from AVHRR and wind in the Greenland Sea", this issue. 